\title{
Microlens array imaging system for photolithography
}

\author{
Reinhard Völkel \\ Hans Peter Herzig \\ Philippe Nussbaum \\ Rene Dändliker \\ IMT Neuchâtel \\ Rue A.-L. Breguet 2 \\ CH-2000 Neuchâtel, Switzerland \\ E-mail: voelkel@imt.unine.ch \\ William B. Hugle \\ Hugle Lithography \\ Costa di Dentro 2 \\ $\mathrm{CH}-6614$ Brissago, Switzerland
}

\begin{abstract}
A micro-optical system is proposed that uses a stack of four microlens arrays for 1:1 imaging of extended object planes. The system is based on the concept of multiple-aperture imaging. A compact system is presented that is remarkable in that it provides a diffraction-limited resolution of $3 \mu \mathrm{m}$ for unlimited object and image areas. Resolution of 5 $\mu \mathrm{m}$ has been demonstrated for an area of $20 \times 20 \mathrm{~mm}^{2}$ in an experimental setup using melting resist microlens arrays (190- $\mu \mathrm{m}$ lens diameter). The investigated imaging system was developed in connection with a new contactless photolithographic technique called microlens lithography. This new lithographic imaging technique provides an increased depth of focus $(>50 \mu \mathrm{m})$ at a larger working distance $(>1 \mathrm{~mm})$ than with customary proximity printing. Potential applications are photolithography for large print areas (flat panel displays, color filters), for thick photoresist layers (micromechanics), on curved surfaces (or substrates with poor planarity), in V grooves, etc. (c) 1996 Society of Photo-Optical Instrumentation Engineers.
\end{abstract}

Subject terms: micro-optics; microlens arrays; multiple aperture; photolithography; flat-panel display fabrication; micromechanics.

Paper 37036 received Mar. 25, 1996; accepted for publication June 7, 1996.

\section{Introduction}

The investigated microlens array imaging system was developed in connection with a new contactless photolithographic technique called microlens lithography. ${ }^{1,2}$ This new technique is aimed at flat panel displays, micromechanics, or multichip module manufacturing that requires only moderate resolution, on the order of 3 to $5 \mu \mathrm{m}$. The goal is to create an erect 1:1 image of a large lithographic mask on a photosensitive layer. The microlens imaging system will be integrated into proximity printing machines.

Conventional imaging systems, such as lithographic or photographic objectives, consist of different optical elements in a linear arrangement. The image is transported within a single optical channel. All elements have a common optical axis. The optical properties are defined by aperture and focal length. Array imaging systems transport the light through different optical channels in parallel-for example, through an array of microlenses, objectives, or telescopes. The image formation can be completely separate for each channel (fly's eye, integral photography), or images transported by adjacent channels can overlap or interfere to form complex composite images. Now, the optical properties depend on the parameters of the local subsystems, on the geometry of the array, and on the optical properties of the illumination.

Array imaging systems using multiple apertures have been widely studied for many decades. ${ }^{3-11}$ Examples of applications include photocopying machines, ${ }^{12,13}$ cameras, ${ }^{14}$ integral photography, ${ }^{15,16}$ scanners and printers, ${ }^{17}$ optical correlators, ${ }^{18}$ and multiple-mirror telescopes. ${ }^{19,20}$ In the past, the resolution of microlens array imaging systems was mostly limited to a few lines per millimeter and rarely suitable for lithographic applications. A significant im- provement of the optical performance can be achieved by using melting resist microlens arrays and an optimized symmetrical setup based on the system proposed by Anderson. ${ }^{8}$

\section{Description of the Microlens Array System}

The described system is a multiple-aperture system consisting of a stack of four microlens arrays forming an array of micro-objectives. A scheme of two adjacent microobjectives is shown in Fig. 1. Two microlens arrays L1 and L2 are used for imaging. Two arrays FL1 and FL2 are combined to serve as a field lens array. The setup is fully symmetrical. A demagnification of the intermediate image

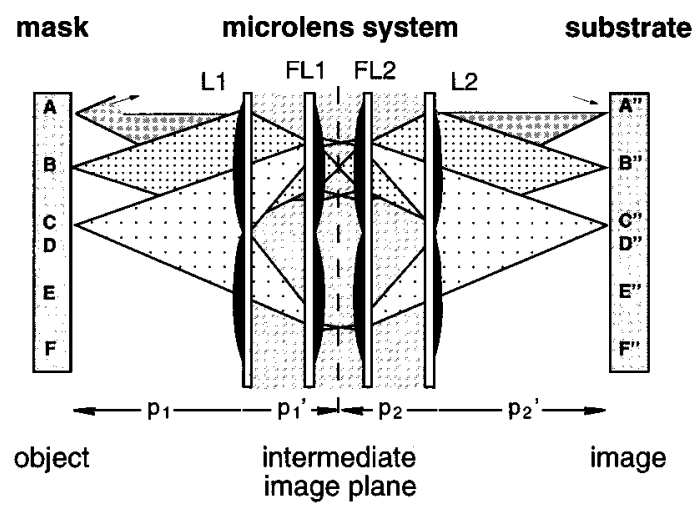

Fig. 1 Microlens array system for erect imaging at unit magnification. Two microlens arrays, L1 and L2, are used for imaging. A combination of two microlens arrays, FL1 and FL2, serves as a field lens. 


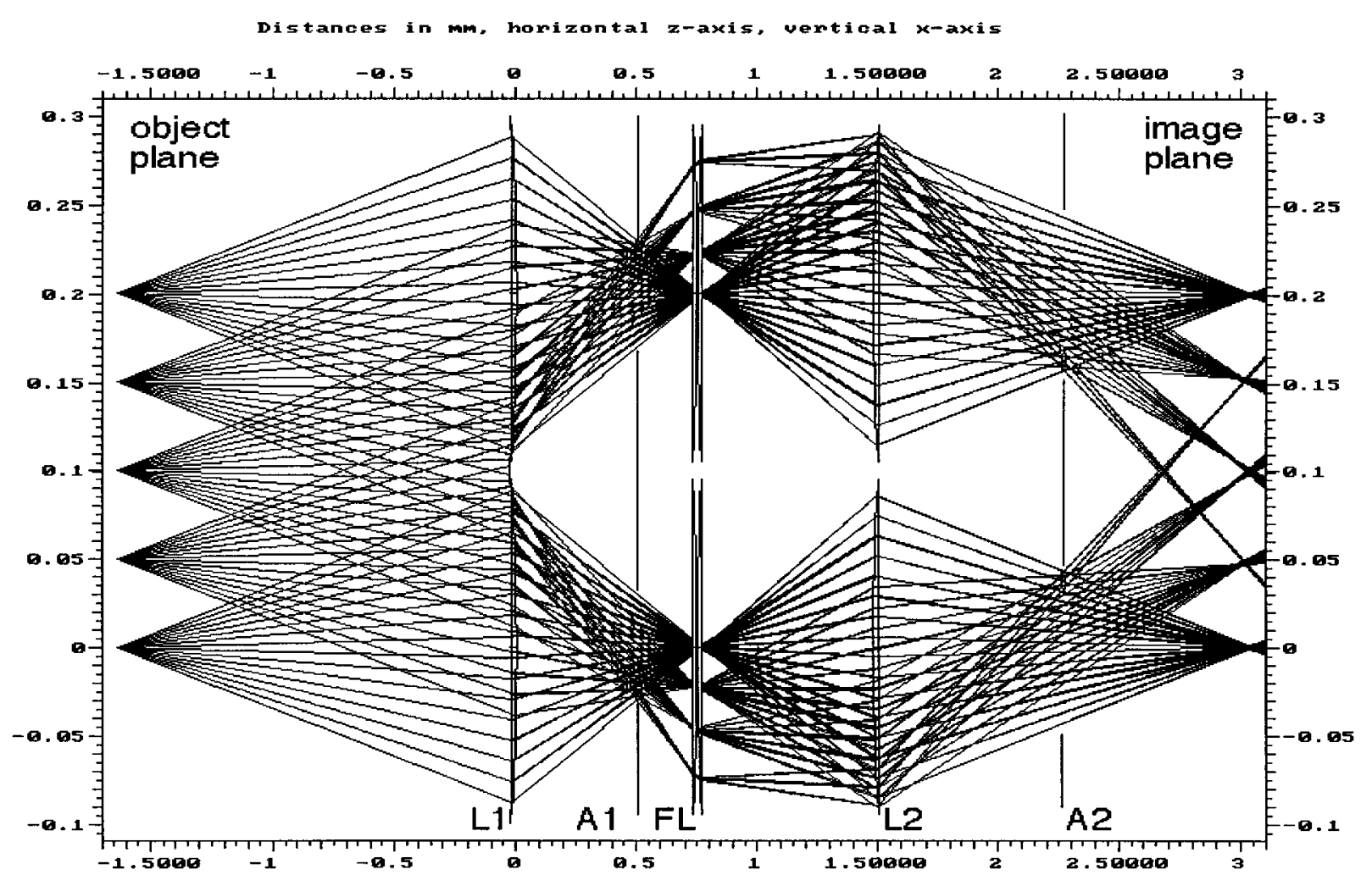

Fig. 2 Ray-tracing analysis for two adjacent micro-objective channels. The images overlap in the image plane. A diffraction-limited resolution of $3 \mu \mathrm{m}$ was found for the whole image field.

is introduced to avoid crosstalk between adjacent microobjective channels. ${ }^{8}$ Each point in the object plane is simultaneously transported by different micro-objectives. The partial images overlap coincidentally in the final image to provide a single, complete image of the object.

Figure 2 shows ray tracing for two adjacent microobjective channels. The system was calculated for refractive microlenses (plano-convex asphere; 198- $\mu \mathrm{m}$ diam; fused silica, $n_{406}=1.469$; focal lengths $f_{\mathrm{L} 1,2}=500 \mu \mathrm{m}$ and $\left.f_{\mathrm{FL} 1,2}=750 \mu \mathrm{m}\right)$. The images are transported by different channels and overlap perfectly in the image plane. A diffraction-limited spot size of $3 \mu \mathrm{m}\left(\mathrm{NA} \approx 0.07, \lambda_{h}=406\right.$ $\mathrm{nm})$ was found for the whole image field. Figure 2 shows the distribution of the rays in the image plane.

The imaging system takes advantage of the following optical design concepts:

1. Symmetry: For symmetrical systems the antisymmetrical wavefront aberrations (coma, distortion, and lateral color) are minimized. ${ }^{21,22}$ However, symmetrical wavefront aberrations (spherical aberration) are doubled and have to be compensated in another way.

2. Aspherical surfaces: Spherical aberration can be minimized by using aspherical lenses. A planoconvex lens profile is generally described by

$h(r)=\frac{1}{R} \cdot \frac{r^{2}}{1+\left[1-(K+1) r^{2} / R^{2}\right]^{1 / 2}}+$ higher terms,

wherein $h$ is the height of the lens as a function of the distance $r$ to the optical axis, $R$ is the radius of curvature at the vertex, and $K$ is the aspherical constant. A plano-convex hyperboloid $\left(K=-n^{2}\right.$, wherein $n$ is the refractive index) has no spherical aberration for the paraxial region. ${ }^{23}$

3. Scaling law: A downscaling of the diameter $D$ and the focal length $f$ of a lens improves its optical performance. ${ }^{24}$ For a fixed stop number $F=f / D$, the diffraction-limited resolution is given by $\delta x \approx \lambda F$. The depth of focus is given by $\delta z \approx 4 \lambda F^{2}$. Both values are independent of the lens scale. The wave aberrations describe the deviation of the actual wavefront from a perfect spherical wavefront. Wave aberrations are usually expressed in fractions of the wavelength. A scaling of all length parameters of a lens does not affect the shape of the wavefront. However, a scaling changes the magnitude of wavefront aberration. Small lenses have less aberrations than large lenses (for the same $F$ number and wavelength). On the other hand, small lenses have a shorter focal length, and thinner lens base plates are required to keep the focal plane outside the base plate. Manufacturing, packaging, alignment, and stability aspects limit downscaling of the microlenses.

4. Telecentry: For telecentric systems a small defocusing by changing the distance of the object or the observing plane does not affect the image size. Systems can be frontal telecentric, rear telecentric, or both. Frontal telecentric systems have a stop in the back 


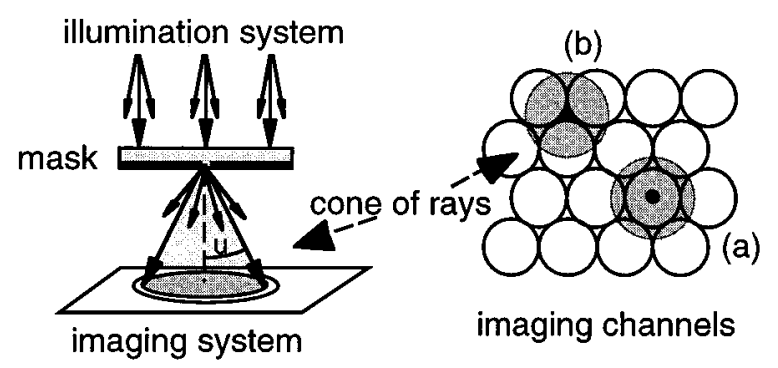

Fig. 3 The distribution of the light at the entrance pupil of the imaging system is governed by the illumination of the object plane and the diffraction at the object.

focal plane of the lens; rear telecentric systems have a stop in the front focal plane. The described system (Fig. 2) is frontal telecentric. An aperture A1 is placed in the back focal plane of L1 and imaged by FL1, FL2, and L2 into plane A2. Plane A2 is situated in the middle between L2 and the image plane. The system is not rear telecentric. An additional lens placed near the image plane could provide rear telecentry, but that would not make much sense for the lithographic application. The partial telecentric behavior of the system ensures that a slight defocusing influences only the spot size and does not change the lateral position of the spot center.

5. Multiple apertures: Telescope arrays are well known in astronomy to enhance the collecting capacity and the resolving power. ${ }^{25,26}$ Instead of using a large mirror, multiple-mirror telescopes are formed by an array of smaller mirrors. Each mirror produces its own diffraction image. When these images superimpose, their amplitudes add to form the final image. When the different images of one object point superimpose in phase (coherent telescope), the resolving power is increased. In astronomy, an object normally consists of several adjacent stars (small luminous points) at infinity. Multiple telescope systems can be adjusted to a fraction of the wavelength to ensure constructive interference in the image plane. This cannot be done for a multiple-lens system as shown in Fig. 2. The optical path length varies for different object points and channels. The image information might be partially lost if the images interfered destructively. Therefore, incoherent image formation is mandatory, and the resolving power is not enhanced. The proposed system takes advantage of the fact that the image quality is invariable for the whole image field. The image size is only limited by the size of the microlens array system. No matter how large the system is, it will provide uniform image quality (no field curvature, distortion, or contrast degradation at the edge of the image).

\section{Imaging Properties}

The described microlens system projects a planar object on an image plane located some 1 to $2 \mathrm{~mm}$ behind the system (see Fig. 2). The object is illuminated from the back side (see Fig. 3). The angular spectrum behind the object is determined by the aperture of the illumination and by the diffraction at the object.

An object is resolved if the optical system is able to transmit at least two diffraction orders generated by the object (Abbe theory). The fidelity of the image increases if more spatial frequencies are allowed to pass the lens system. However, for photolithography two orders are sufficient to define useful patterns, because of the high contrast of the photoresist. ${ }^{27}$

The maximum angle transmitted in the microlens array system is limited by vignetting effects within the imaging channels. To obstruct stray light and crosstalk between adjacent channels, all image-forming rays that pass the entrance pupil (lens L1) of an imaging channel must pass the exit pupil (lens L2) of the same channel (see Fig. 2). For this purpose, a stop array A1, located in the back focal plane of the lens array L1, is used as field stop. The angular field diameter is limited to an angle of $u \approx \pm 4 \mathrm{deg}$ for the proposed setup. Thus, the numerical aperture is $\mathrm{NA}=\sin u$ $\approx 0.07$, and the diffraction-limited resolution is $\delta x \approx 3 \mu \mathrm{m}$ for incoherent imaging using UV light at $\lambda=406$-nm wavelength. We used microlens arrays consisting of circular lenses in a hexagonal close-packed arrangement. The fill factor $\eta$ is given by

$\eta=\frac{\emptyset^{2} \pi}{2 a^{2} \sqrt{3}}$,

which yields $\eta \approx 88 \%$ for a lens diameter of $\emptyset=198 \mu \mathrm{m}$ and a lens pitch of $a=200 \mu \mathrm{m}$. The space between the lenses is blocked (e.g., by a thin chromium layer). For this geometry, all object points are less than $100 \mu \mathrm{m}$ away from the nearest local optical axis. The number of contributing channels for the transmission of a single object point is determined by the aperture of the system, the distance $p_{1}$ between the object plane and the entrance pupils, and the diameter $\emptyset$ of the entrance pupils. A distance of $p_{1} \approx 3 f_{\mathrm{L} 1}$ leads to a demagnification of the intermediate image by a factor $m_{1}=p_{1}^{\prime} / p_{1} \approx 0.5$ (see Fig. 1 ). The number of contributing channels increases with demagnification of the intermediate image. On the one hand, a large number of contributing channels improves the uniformity of the image; on the other hand, it reduces the achievable resolution because the aberrations are increasing. A good compromise is to use six or seven adjacent channels as indicated in Fig. 3. This leads to a uniformity of the image intensity on the order of $\pm 3 \%$ for ideal illumination.

\section{Illumination}

The significant influence of the illumination properties on the image formation has already been discussed in the previous sections. In practice, we take advantage of the very high standard of illumination systems used in photolithographic machines. The illumination system of a standard proximity printer (e.g., SUSS MA150 from Karl Suess KG, Munich, FRG) provides partially incoherent collimated light from a mercury lamp ( $g, h$, or $i$ line). The collimation angle is usually adjustable from 1 to $4 \mathrm{deg}$. Such illumination systems are well suited for the proposed microlens array imaging system. The collimation angle can be adapted to the numerical aperture of the imaging system. A further improvement of the system properties is expected by adding diffusers, microlens arrays, gratings, or 


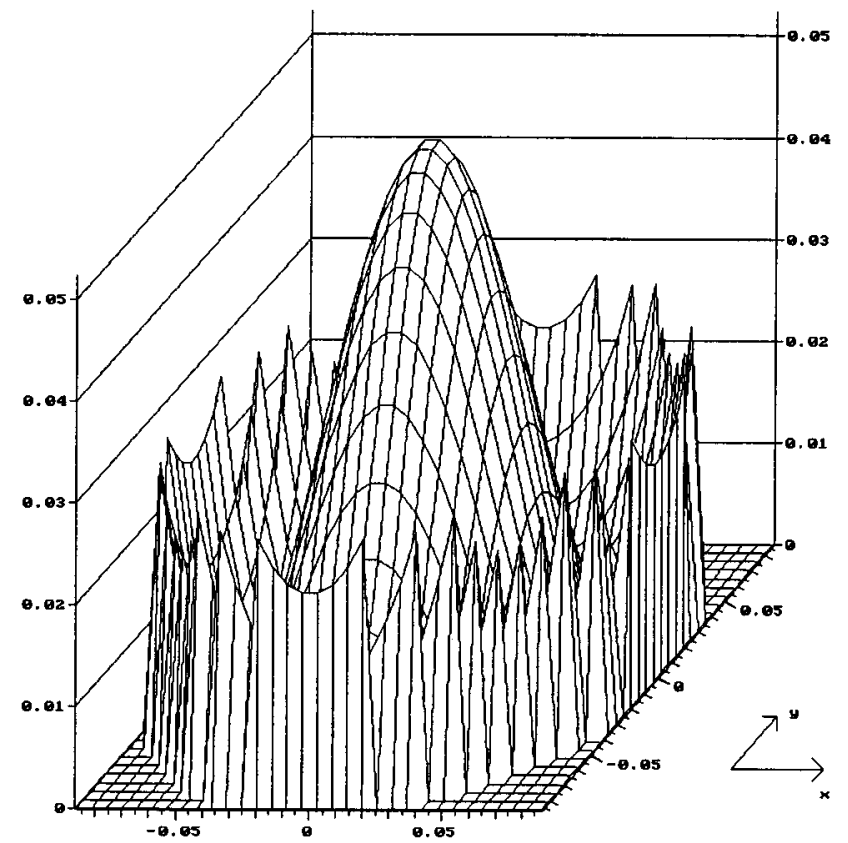

(a)

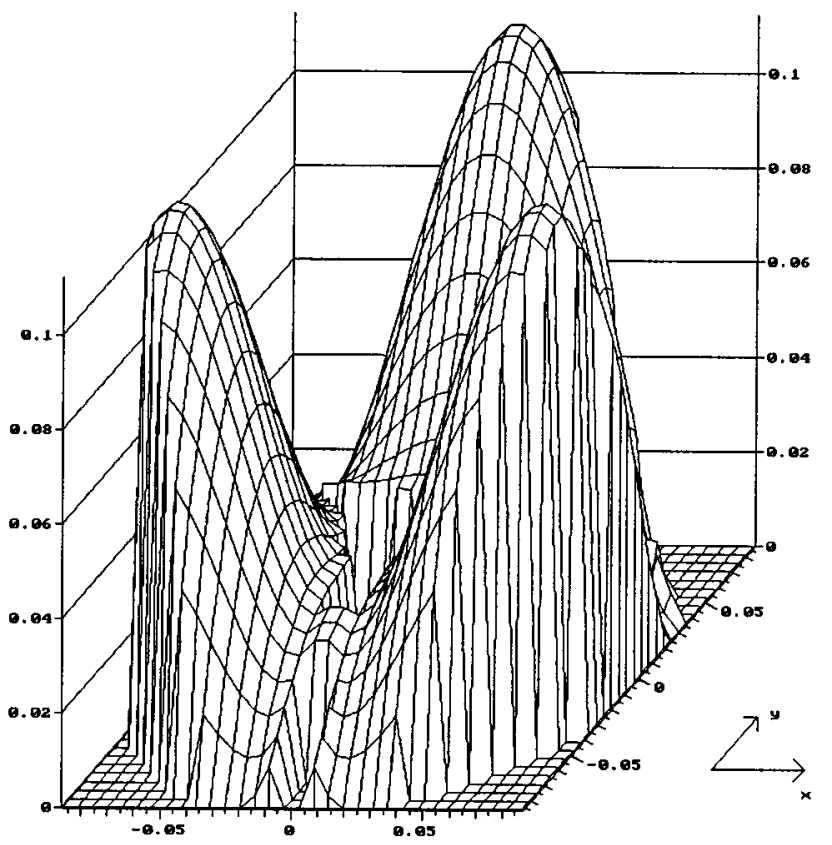

(b)

Fig. 4 Wavefront deviation for the imaging system shown in Fig. 2. Multiple apertures were used for imaging an object point that is located (a) on the optical axis of one lens and (b) at the center of three adjacent lenses, as indicated in Fig. 3 (right).

computer-generated holograms to manipulate the illuminating light. Nonuniformity of the image intensity could be compensated. Nonconventional lithographic techniques such as off-axis lithography could be implemented using array imaging systems.

\section{Image Contrast and Resolution}

Photolithographic imaging systems are characterized by the diffraction-limited resolution $\delta x$ and the depth of focus $\delta z$, which are in general given by

$\delta x=K_{1} \frac{\lambda}{\mathrm{NA}}$,

$\delta z=K_{2} \frac{\lambda}{\mathrm{NA}^{2}}$,

where $\lambda$ is the wavelength and NA is the numerical aperture. Here $K_{1}$ and $K_{2}$ are scaling factors that are determined by the lithography process. ${ }^{27}$ The factor $K_{1}$ has been reduced from about 1.0 to below 0.7 , mainly by resist and processing improvements. Using incoherent illumination, $K_{1}$ is below 0.5 . The factor $K_{2}$ is 1.0 for classical imaging systems.

The irradiance of an aberrated diffraction image spot at the center is called the Strehl ratio. ${ }^{22}$ The Strehl ratio is a function only of the wavefront variance, or the square of the rms wavefront deviation. Figure 4 shows the wavefront deviation for imaging (a) on-axis and (b) off-axis object points, as indicated in Fig. 3. The calculation of the wavefront deviation is based on ray tracing. Strehl ratios of 0.99 and 0.96 were found for (a) and (b). The Strehl ratios cor- respond to rms wave aberrations smaller than $\lambda / 20$. From the Maréchal criterion it is known that aberrations will not degrade the image if the rms wavefront error is smaller than $\lambda / 14$.

The modulation transfer function (MTF) gives the image contrast as a function of the spatial frequency of the object. The MTF is the autocorrelation of the pupil function for incoherent imaging systems. ${ }^{21,28}$ Figure 5 shows the MTF for (a) on-axis and (b) off-axis imaging as indicated in Fig. 3. The calculation of the MTF is based on ray-tracing analysis. An image contrast of 0.3 is found for a resolution of $5 \mu \mathrm{m}$ (or 100 lines $/ \mathrm{mm}$ ). Ray-tracing analysis of the proposed setup confirms diffraction-limited optical performance over the whole image field.

\section{Chromatic Aberration}

The proposed array imaging system was investigated for a wavelength of $\lambda_{h}=406 \mathrm{~nm}$, the $h$ line of a mercury lamp. In the following we want to analyze the chromatic properties of the system.

For multiple-pupil systems, the formation of a final (or composite) image is governed by two fundamental mechanisms: the imaging, and the overlap of the images transported through different micro-objective channels. The imaging is governed by the optical properties of the individual channels. A change of the wavelength will change the focal length of the lenses (due to dispersion) and will change the position of the image plane. Anderson ${ }^{8}$ points out that the position of the so-called plane of coincidence is only a property of symmetry and independent of the power of the lenses for fully symmetrical systems. Therefore, a change of the wavelength does not affect the overlap of the images, 


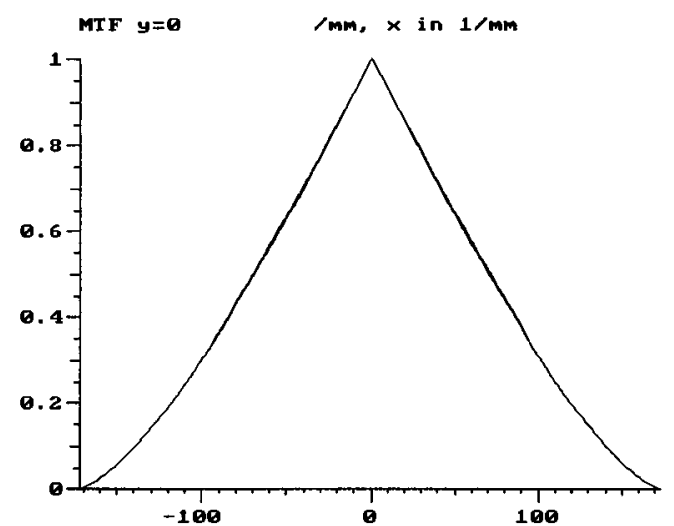

(a)

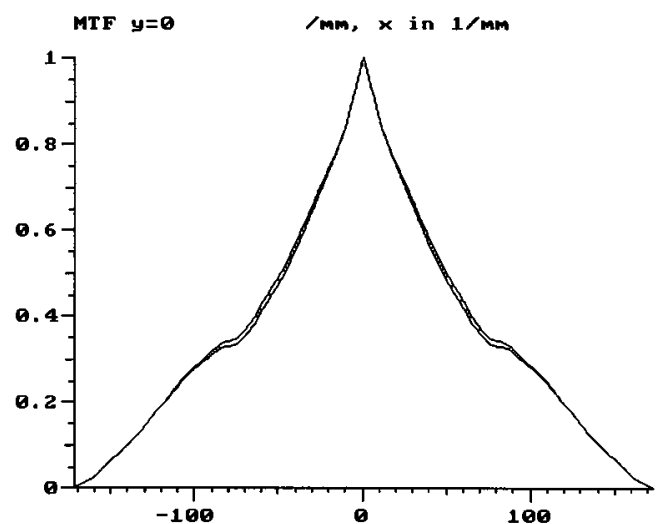

(b)

Fig. 5 Modulation transfer function (MTF) calculated from the wavefront deviation shown in Fig. 4.

but it will defocus the images in the plane of coincidence. The mismatch between the two planes can be corrected by changing the object position. Each wavelength requires a different object position. Multiple-wavelength imaging is accompanied by a reduction of the resolution in the composite image.

The proposed system was analyzed for multiplewavelength imaging with three mercury lines $\left(\lambda_{i}=365 \mathrm{~nm}\right.$, $\left.\lambda_{h}=406 \mathrm{~nm}, \lambda_{g}=436 \mathrm{~nm}\right)$. The object position was optimized for the $h$ line. Image shifts of $\Delta p_{2}^{\prime}\left(\lambda_{i}\right) \approx-30 \mu \mathrm{m}$ and $\Delta p_{2}^{\prime}\left(\lambda_{g}\right) \approx+20 \mu \mathrm{m}$ were found for the $i$ and the $g$ line. A significant reduction of the resolution is observed in the composite image. A resolution on the order of $\delta x=6$ to $8 \mu \mathrm{m}$ and a usable depth of focus on the order of $\delta x=100$ to $150 \mu \mathrm{m}$ are found for multiple-wavelength illumination with two or three mercury lines.

\section{Microlens Arrays}

The most critical point for the realization of the proposed imaging system is the availability of suitable microlens arrays. Microlens arrays can be subdivided into three categories: (1) diffractive lenses, (2) refractive lenses (e.g., melting resist lenses), and (3) gradient index (GRIN) lenses.

Diffractive lenses offer full freedom of wavefront design, a $100 \%$ fill factor, and excellent reproducibility of all lens parameters. Unfortunately, the focal length is strongly dependent on the wavelength, and the use of diffractive lenses is limited to monochromatic applications. Highquality diffractive microlens arrays are available now. However, diffractive lenses still generate a fair amount of undesired scattering or stray light, on the order of 5 to $8 \%$ of the incident light (spurious orders, ghost images, edge effects, etc.). A stack of four diffractive microlens arrays for the proposed system design ( $F$ number 2 and 4$)$ would suffer from severe stray-light problems, poor total efficiency (the diffraction efficiency is $\eta_{\mathrm{tot}}=\eta_{1} \eta_{2} \cdots$ ), and chromatic aberration. A combination of refractive and diffractive lenses (hybrid system) could be used for monochromatic imaging.

Planar GRIN lenses are excellent for imaging; unfortunately, they are not transparent in the UV.

A promising candidate for the implementation of the investigated imaging system is the melting resist technology. ${ }^{29,30}$ Photoresist cylinders are formed by lithography and melted at a temperature $T \approx 150^{\circ} \mathrm{C}$. Surface tension forms plano-convex spherical microlenses. The focal length is given by

$f=\frac{h_{L}+r^{2} / h_{L}}{2(n-1)} \approx \frac{r^{2}}{h_{L}}$,

where $r$ is the lens radius and $h_{L}$ is the height of the lens. Melting resist microlenses are well suited for highresolution imaging. Figure 6 shows a test pattern imaged with a single melted resist lens. A resolution of $1.2 \mu \mathrm{m}$ is observed in a microscope with white-light illumination.

The photoresist itself is not transparent in the UV. The microlens array is transferred to fused silica by reactive-ion etching (RIE) ${ }^{31-33}$ Profile shaping is possible by varying the RIE process. A change of the reactive-gas content influences the etch ratio between resist and silica during the RIE process. This effect is used to correct the slope of the lens in the rim region and to reduce spherical aberration. Aspherical microlenses have been fabricated recently using this technology. ${ }^{34,35}$ A different approach is preshaping of the resist cylinder by using multilevel gray-level mask technology or direct writing, ${ }^{36,37}$ and subsequent melting to smooth the resist surface before the microlenses are etched in fused silica.

The uniformity of a microlens arrays is determined by the accuracy of the resist cylinders, the melting process, and the RIE transfer to fused silica. The described technology requires only standard semiconductor techniques for

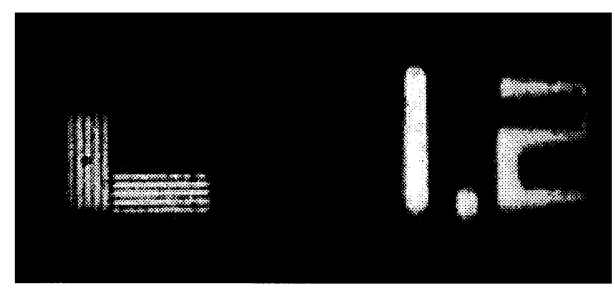

Fig. 6 Test pattern imaged with a single melting resist microlens $(\Phi=250 \mu \mathrm{m}, \mathrm{NA}=0.36)$. A resolution of 400 lines $/ \mathrm{mm}(1.2-\mu \mathrm{m}$ feature size) is observed in a microscope under white-light illumination. 


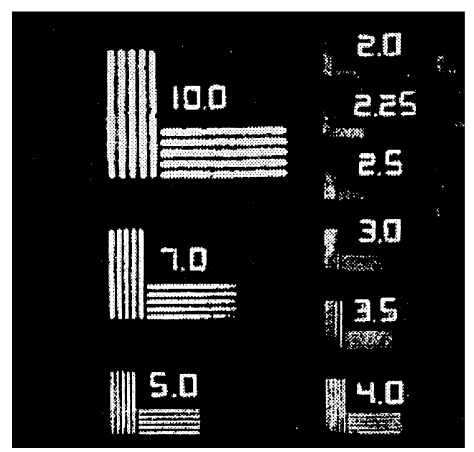

Fig. 7 Image plane of a multiple aperture imaging system observed in a microscope under white-light illumination. A resolution of $5 \mu \mathrm{m}$ is observed.

resist coating and processing, lithography, and RIE. Melting resist microlens arrays with uniformity of the focal length better than $1 \%$ have been be demonstrated recently. ${ }^{38}$ Since this technology has been investigated solely within research departments, no reliable values for uniformity and reproducibility are available for mass production in an industrial environment.

\section{Preliminary Demonstration System}

A $20 \times 20-\mathrm{mm}^{2}$ demonstration system for preliminary resolution tests has been built. The experimental setup differed slightly from Fig. 2. Two arrays of spherical plano-convex melting resist lenses $\left(\emptyset=190 \mu \mathrm{m}, f_{\mathrm{L} 1} \approx 600 \mu \mathrm{m}\right)$ were used for imaging. One array of microlenses $(\emptyset=190 \mu \mathrm{m}$, $f_{\mathrm{L} 1} \approx 400 \mu \mathrm{m}$ ) served as field lens. Spacer elements (glass balls, fiber pieces) were used for the vertical alignment of the lens arrays. The horizontal alignment was done by hand under a microscope. The moiré effect between identical patterns allows a precise lateral alignment of the microlens arrays.

The image plane was observed with a microscope. Figure 7 shows the image plane of a standard resolution target. A resolution of $5 \mu \mathrm{m}$ for the whole image field has been observed for white-light illumination. Figure 8 shows the image of a mask pattern as used for flat-panel-display fabrication.

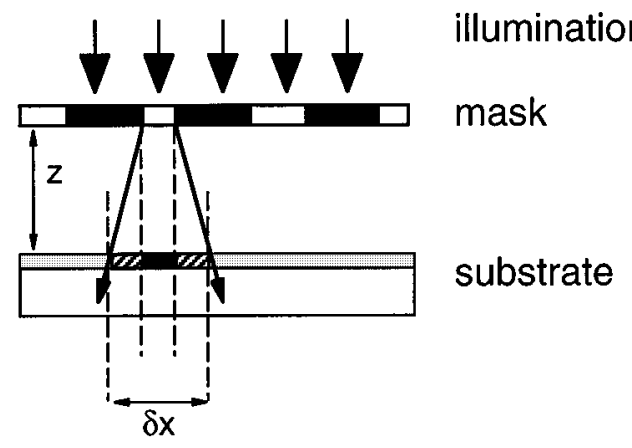

Fig. 9 Proximity printing. The resolution $\delta x$ decreases rapidly with increasing distance $z$ between the mask and the substrate.

\section{Applications}

The proposed array imaging system was developed in connection with a new photolithographic technique called $\mathrm{mi}$ crolens lithography. Figure 9 shows schematically the mask pattern transfer within a proximity printer. Figure 10 shows the image formation for microlens lithography. There are two basic advantages of this new lithographic technique compared to standard proximity printing:

- Large working distance: The distance between substrate and imaging system is on the order of $z=1 \mathrm{~mm}$ (Fig. 10), which is much larger than for proximity printing with $z=20$ to $50 \mu \mathrm{m}$ (Fig. 9). There is no risk of damaging mask or substrate. Lithography is feasible in holes, in grooves, or on other surfaces where close contact is not possible.

- Large depth of focus: For proximity printing, the resolution decreases rapidly with increasing distance $z$ (Fig. 9). For microlens lithography, the best resolution is observed in the image plane. The image is extended forward and backward (Fig. 10). A resolution of $\delta x \approx 5 \mu \mathrm{m}$ corresponds to a usable depth of focus of $\delta z>50 \mu \mathrm{m}$ for microlens lithography.

For proximity printing, the substrate and alignment costs (surface planarity) increase rapidly with the resolution. The larger depth of focus at a noncritical working distance offers a tremendous potential to cut fabrication costs for lithography in the 5- to $10-\mu \mathrm{m}$ resolution domain. Potential
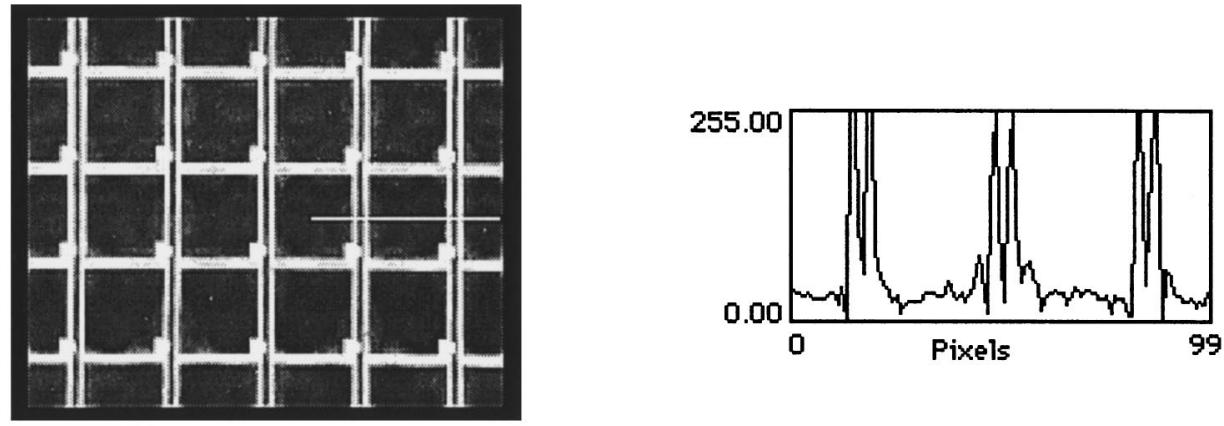

Fig. 8 Image of a test pattern as used for flat-panel-display fabrication. No distortion or magnification errors are observed. 
Völkel et al.: Microlens array imaging system ...

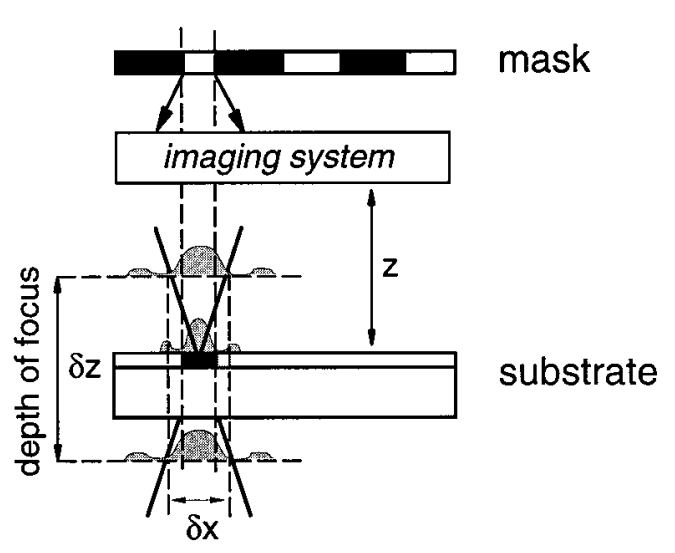

Fig. 10 Microlens lithography. The image plane is located at a distance $z$ behind the imaging system. There is no risk of damaging the mask or the substrate. The image is extended both forward and backward. The depth of focus is given by $\delta z \approx \lambda / N A^{2}$.

applications for microlens lithography are photolithography on thick photoresist layers, curved surfaces, and $V$ grooves or over very large print areas. Moreover, the proposed microlens imaging system might be very useful for inspection systems, neural networks, optical interconnects, etc. Such miniaturized 1:1 imaging systems could be used to connect receiver or detector arrays (CCDs, photodiodes, fibers, smart pixels) with light sources (laser, LED), fibers, or pixel arrays. Diffractive optical elements or active matrix elements (LCDs, light valves) could be integrated into the imaging system to implement filtering, switching, splitting, or integrating operations.

\section{Conclusion}

A multiple-aperture microlens system for the 1:1 imaging of extended objects has been proposed. A compact system design has been presented, which is remarkable in that it provides a diffraction-limited resolution of $3 \mu \mathrm{m}$. The system design has been analyzed by ray tracing. A preliminary demonstration system using melting resist microlenses has been built. A resolution of $5 \mu \mathrm{m}$ has been demonstrated experimentally for a $20 \times 20$-mm image field and whitelight illumination.

This publication summarizes the first step in developing a novel lithographic technique. The reported theoretical and experimental results have attracted broad attention within the display and semiconductor industry. Of course, there are still significant manufacturing and alignment problems that have to be solved. But the chance to cut fabrication and substrate costs by using this new lithographic technique is a strong stimulus for future investigations.

\section{Acknowledgment}

This project was partially supported by Hugle Lithography, Karl Suss KG, and the Swiss Priority Program "Optique." The authors thank S. Haselbeck, M. Eisner, and J. Schwider for their help in the microlens fabrication and characterization, and E. Cullmann (Suss) and M. Hutley (NPL) for helpful discussions.

\section{References}

1. W. B. Hugle, R. Daendliker, and H. P. Herzig, "Lens array photolithography," G. B. Patent Appl. 9224080.3 (1992), U.S. Patent No. 08/114,732 (1993)

2. R. Dändliker, S. Gray, F. Clube, H. P. Herzig, and R. Völkel, "Nonconventional fabrication methods for photolithography," in $M N E$ Micro- and Nano-Eng. '94, Davos Sept. 26-29 (1994).

3. G. C. Lippmann, "Eppeuves reversibles donnant la sensation du relief," J. Phys. (Paris) 821 (1908).

4. H. E. Ives, "Optical properties of a Lippmann lenticulated sheet," $J$. Opt. Soc. Am. 21, 171 (1931).

5. D. Gabor, U.K. Patent No. 541.753 (1940).

6. H. Käufer, E. Burger, and H. P. Huber, FRG Patent No. 1.260.178 (1968); U.S. Patent No. 3.447.438 (1969)

7. I. Kitano, K. Koizumi, H. Matsumura, K. Ikeda, and T. Uchida, "Image transmitter formed of a plurality of graded index fibers in bundled configuration,' U.S. Patent No. 3,658,407 (1972).

8. R. H. Anderson, "Close-up imaging of documents and displays with lens arrays," Appl. Opt. 18, 477-484 (1979).

9. I. Glaser and A. A. Friesem, in Application of Holographic and Optical Data Processing, E. Moram, Ed., Pergamon Press (1977).

10. I. Glaser-Inbari, "Optical imaging system," U.S. Patent No. 4,175,844 (1979).

11. N. F. Borelli, R. H. Bellman, J. A. Durbin, and W. Lama, "Imaging and radiometric properties of microlens arrays," Appl. Opt. 30, 3633$3642(1991)$.

12. M. Kawazu and Y. Ogura, "Application of gradient-index fiber arrays to copying machines," Appl. Opt. 19, 1105-1112 (1980).

13. K. Hamanaka, H. Nemoto, M. Oikawa, E. Okuda, and T. Kishimoto, "Multiple imaging and multiple Fourier transformation using planar microlens arrays," Appl. Opt. 29, 4064-4070 (1990).

14. R. A. Anderson, "Optical apparatus including a pair of mosaics of optical imaging elements," U.S. Patent No. 3,605,593 (1971).

15. N. Davies and M. McCormick, "Three dimensional imaging systems: a new development,' Appl. Opt. 27, 4520-4528 (1988).

16. M. Hutley and R. F. Stevens, "The formation of integral images by a focal pairs of lens arrays ('superlenses')," in IOP Short Meetings Series No. 30, ISBN 0-85498-531-X, pp. 147-154 (1991).

17. R. W. Gundelach, "Lens strip optical scanning system," U.S. Patent No. 3,584,950 (1971).

18. I. Glaser, "Non-coherent parallel optical processor for discrete twodimensional linear transformations," Opt. Lett. 5, 449-451 (1980).

19. A. B. Meinel, "Aperture synthesis using independent telescopes," Appl. Opt. 9, 2501-2504 (1970).

20. R. V. Shack, J. D. Rancourt, and H. Morrow, "Effects of dilution on a six-element synthetic aperture," Appl. Opt. 10, 257-259 (1971).

21. R. Kingslake, Lens Design Fundamentals, Academic Press, New York (1978).

22. D. Malacra and Z. Malacara, Handbook of Lens Design, Marcel Dekker (1994).

23. H. Naumann and G. Schroeder, Bauelemente der Optik, Carl Hanser Verlag, Munich (1992).

24. A. W. Lohmann, "Scaling laws for lens systems," Appl. Opt. 28 , 4996-4998 (1989)

25. A. B. Meinel, M. P. Meinel, and N. J. Woolf, "Multiple aperture telescope diffraction images," in Applied Optics and Optical Engineering, R. R. Shannon and J. C. Wyant, Eds., Vol. IX, Chap. 5, Academic Press, San Diego (1983).

26. Ch. B. Hogge, J. F. Schultz, D. B. Mason, and W. E. Thompson, "Physical optics of multiaperture systems," Appl. Opt. 27, 51275134 (1988)

27. S. Wittekoek, "Optical lithography: present status and continuation below 0.25 nm," Microelectron. Eng. 23, 43-55 (1994).

28. C. Hofmann, Die Optische Abbildung, Akademische Verlagsgesellschaft, Leipzig (1980).

29. Z. D. Popovic, R. A. Sprague, and G. A. Neville-Connell, "Technique for the monolithic fabrication of microlens arrays," Appl. Opt. 27, 1281-1284 (1988)

30. D. Daly, R. F. Stevens, M. C. Hutley, and N. Davies, "The manufacture of microlenses by melting photoresist," J. Meas. Sci. Technol. 1 759-766 (1990)

31. M. Eisner, S. Haselbeck, H. Schreiber, and J. Schwider, "Reactive ion etching of microlens arrays into fused silica," EOS Topical Meet ing Digest Ser. 2, 17-19 (1993).

32. E. Gratix, "Evolution of a microlens surface under etching conditions," Proc. SPIE 1992, 266-274 (1993).

33. M. B. Stern and T. R. Jay, "Dry etching for coherent refractive microlens arrays," Opt. Eng. 33, 3547-3551 (1994).

34. M. Eisner and J. Schwider, "Transferring resist microlenses into silicon by RIE,', Opt. Eng. (in press).

35. K. Mersereau, C. R. Nijander, W. P. Townsend, and A. Y. Feldblum, "Aberration-corrected etched microlens arrays, " in Technical Digest of the Topical Meeting on "Diffractive Optics and Micro-Optics," OSA, Boston (Apr. 1996). 
36. M. T. Gale and K. Knop, "The fabrication of fine lens arrays by laser beam writing," Proc. SPIE 398, 347-353 (1983).

37. E.-B. Kley, T. Possner, and R. Göring, "Realization of micro-optics and integrated optic components by electron-beam-lithographic surface," Int. J. Optoelectron. 8, 513-527 (1993).

38. P. Greiner, "Différentes méthodes de caractérisation de microlentilles," Travail de stage, Université de Neuchâtel, Switzerland (1995)

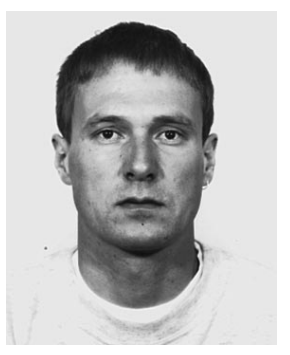

Reinhard Völkel received the diploma in physics in 1989 and the PhD in 1994, both from the University of ErlangenNuernberg, Germany. He joined the Institute of Microtechnique of the University of Neuchâtel, Switzerland, in 1994. His current research activities are in micro-optics, imaging systems, photolithography, and sensors.

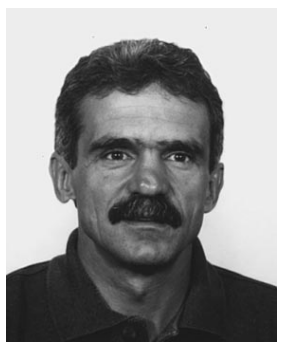

Hans Peter Herzig received the diploma in physics from the Swiss Federal Institute of Technology in Zürich (ETHZ), Switzerland, in 1978. From 1978 to 1982 he was a scientist in the Optics Development Department of the Kern Company in Aarau, Switzerland, where he worked in lens design and optical testing. In 1983, he joined the Applied Optics Group at the Institute of Microtechnology of the University of Neuchâtel, Switzerland, as a graduate research assistant, working in the field of holographic optical elements, especially scanning elements. In 1987, he received the PhD degree in optics from the University of Neuchâtel. Currently, he is lecturing in modern optics and is responsible for the research in micro-optics, including diffractive optical elements and microlenses.

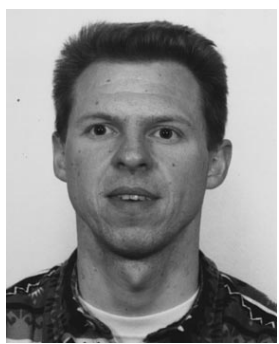

Philippe Nussbaum studied optics in France, where he received his diploma in 1990. In 1992, he was in the Analytical Research Department of the Ciba-Geigy Company in Basel, Switzerland, where he worked on the development of miniaturized optoelectronic sensors for chemical analysis. Since 1993, he has been in the Applied Optics Group at the Institute of Microtechnology of the University of Neuchâtel, Switzerland. He is in charge of the refractive and diffractive micro-optical technology.

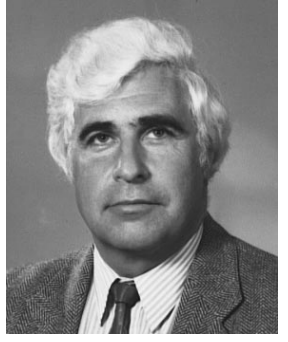

René Dändliker received the diploma in physics from the Swiss Federal Institute of Technology, Zürich, Switzerland, in 1963, the $\mathrm{PhD}$ degree in physics from the University of Berne, Switzerland, in 1968, and the Venia Legendi for applied physics at the Swiss Federal Institute of Technology, Zürich, in 1978. From 1963 to 1969 he was a graduate research assistant at the Institute of Applied Physics, University of Berne, where he worked on gas and solid-state lasers. From 1969 to 1970 he was a research scientist at the Philips Research Laboratories, Eindhoven, Netherlands, in the field of applied optics. From 1970 to 1978 he was a senior scientist and head of the Coherent Optics Group at the Brown Boveri Research Center, Baden, Switzerland, where he was concerned with optical metrology applied to mechanics, such as laser Doppler velocimetry and heterodyne holographic interferometry. Since 1978 he has been a professor of applied optics at the University of Neuchâtel, Switzerland, and since 1989 also professor of applied optics at the Swiss Federal Institute of Technology, Lausanne. His current research activities are in optical metrology, optical fibers and sensors, holography and optical computing, diffractive optical elements, and micro-optics. Dr. Dändliker is president of the European Optical Society (EOS), a fellow of the OSA and the Swiss Academy of Engineering Sciences (SATW), a member of SPIE, the French Society of Optics (SFO), the German Society of Applied Optics (DGaO), and the European Physical Society (EPS), and an affiliate of LEOS/IEEE.

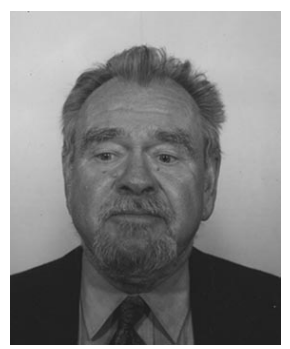

William B. Hugle received the BSc from the University of Chicago in 1947 and the MSc from the University of Cincinnati in 1959, both in physics. He was founder and (vice-)president of Siliconix, Hugle Industries, SEMI, Hugle International, Insystems, and Holtronic Technology. He has worked in the fields of crystalline and semiconductor materials, physical chemistry, holography, FET and IC-fabrication, semiconductor fabrication, and inspection equipment. He is the founder and president of Hugle Lithography. 